\title{
Development of Health-RESPECT: An Integrated Service Model for Older Long-Term Care Hospital/Nursing Home Patients Using Information and Communication Technology
}

Jung-Yeon Choi ${ }^{1}$, Kwang-il Kim ${ }^{1,2}$, Jae-Young Lim ${ }^{3,4,5}$, Jin Young Ko ${ }^{3}$, Sooyoung Yoo ${ }^{6}$, Hongsoo Kim ${ }^{5,7,8}$, Minho Lee ${ }^{9}$, Sae-Kyun Jang ${ }^{10}$, Dong Hee Lee ${ }^{11,12}$, Jungwoo Lee ${ }^{12}$, Young-il Jung ${ }^{13}$, In-Hwan $\mathrm{Oh}^{14}$

\footnotetext{
${ }^{1}$ Department of Internal Medicine, Seoul National University Bundang Hospital, Seongnam, Korea

${ }^{2}$ Department of Internal Medicine, Seoul National University College of Medicine, Seoul, Korea

${ }^{3}$ Department of Rehabilitation Medicine, Seoul National University Bundang Hospital, Seongnam, Korea

${ }^{4}$ Department of Rehabilitation Medicine, Seoul National University College of Medicine, Seoul, Korea

${ }^{5}$ Institute on Aging, Seoul National University, Seoul, Korea

${ }^{6}$ Office of eHealth Research and Businesses, Seoul National University Bundang Hospital, Seongnam, Korea

${ }^{7}$ Department of Public Health Sciences, Graduate School of Public Health, Seoul National University, Seoul, Korea

${ }^{8}$ Institute of Health and Environment, Seoul National University, Seoul, Korea

${ }^{9}$ Healthcare Convergence R\&D Center, ezCaretech Co. Ltd., Seoul, Korea

${ }^{10}$ Healthcare Convergence R\&D Center, Healthconnect Co. Ltd., Seoul, Korea

${ }^{11}$ adelie Co. Ltd., Seoul, Korea

${ }^{12}$ Graduate School of Information and Center for Work Science, Yonsei University, Seoul, Korea

${ }^{13}$ Department of Environmental Health, Korea National Open University, Seoul, Korea

${ }^{14}$ Department of Preventive Medicine, Kyung Hee University School of Medicine, Seoul, Korea
}

Corresponding Author:

Kwang-il Kim, MD, $\mathrm{PhD}$

Department of Internal Medicine,

Seoul National University Bundang

Hospital, Seoul National University

College of Medicine, 82 Gumi-ro

173beon-gil, Bundang-gu, Seongnam

13620, Korea

E-mail: kikim907@snu.ac.kr

ORCID:

https://orcid.org/0000-0002-6658-047X

Received: February 18, 2020

Revised: March 15, 202

Accepted: March 20, 2020
Background: Korea, as one of the fastest-aging countries worldwide, requires an improved healthcare service model for older adults. We evaluated the current healthcare system and developed a service model based on information and communication technologies (ICT) for use in older patients in long-term care facilities (LTCF). Methods: We conducted a qualitative literature review, focus group interviews (FGIs), and structured survey to identify the current technology use and status of healthcare systems. We then developed a web-based platform with necessary, high-priority, and usable content for the care of older patients in LTCF. Results: We reviewed 60 (23 hypertension, 18 diabetes, and 19 heart failure) articles on iinformation and communication technologies (ICT)-based disease management for clinical effectiveness and improved patient satisfaction. FGIs and structured surveys were used to evaluate the inconvenience in patient and medical information transfer between hospitals and cost and time required for its process. Accordingly, we confirmed the unmet need for an ICT-based service model for management, monitoring, and consultation among older patients and developed the Health-RESPECT (integrated caRE Systems for elderly PatiEnts using iCT), a service platform for older patients residing in LTCF. The medical information exchange system was used to transfer medical information. Health-RESPECT includes an established algorithm for evidence-based comprehensive geriatric assessment and customized management; chronic disease management; management of potentially inappropriate medications; rehabilitation; and consultation and videoconferencing. Conclusion: This study identified the current status and unmet needs of healthcare systems for older adults. We developed an ICT-based system to manage older institutionalized patients. However, the Health-RESPECT service model requires further validation.

Key Words: Frailty, Care model, Digital medicine, ICT 


\section{INTRODUCTION}

A "baby boomers" grow older Korea, is one of the fast-aging countries worldwide. In just 17 years, the aging population has doubled from 7\% (an aging society) in 2000 to $14 \%$ (an aged society) in 2017 and is expected to grow to $20 \%$ (a post-aged society) by 2026. ${ }^{1)}$ Older individuals tend to have multiple chronic conditions, poor self-reported health status, and functional dependencies. As a result, older patients are institutionalized in long-term care hospitals (LTCH) or nursing homes (NH). ${ }^{2,3)}$ Thus, the costs of medical care for older adults is expected to increase exponentially, giving rise to the need for alternative, sustainable health and medical systems. However, fragmented medical services in Korea lead to challenges in providing integrated medical services to this population.

The management and care of older adults is a challenge because of their complicated multi-morbidities and variations in their functional, cognitive, and socio-economic statuses. ${ }^{3)}$ In Korea, due to the increase in the number of single-person households and the social activities of women, family support in the care of older adults is decreasing. Consequently, older adults are eventually admitted to LTCH or NH that provide both medical and care support. Particularly in Japan, where the proportion of the aging population is over $27 \%$, long-term care costs have increased more rapidly than medical costs, especially among older adults living alone. ${ }^{4)}$ Thus, we need to respond to the growing demand and expanding costs of healthcare, comprising medical and long-term care costs, in older adults.

To offer sustainable healthcare systems to support active and healthy aging, Europe has developed and validated the Inclusive Introduction of Integrated Care (IN3CA) project as part of the eHealth Action Plan. The project is slated to run from 2012 until 2020 and uses information and communication technologies (ICT) to enable better and more efficient health care at a lower cost and with better continuity of care. The project showed that integrated care with joint care planning, shared clinical records, decision support tools, and care coordination through the use of ICT benefited patient experience, use of services, and costs. ${ }^{5)}$

There is great potential for ICT service platforms to address present and future healthcare and long-term care management of older people. ${ }^{6)}$ ICT can positively affect both intergenerational and partner care by decreasing usability barriers and promoting attractive and collaborative environments for informal care. ICT platforms and communication channels also allow us to avoid duplication of efforts when dealing with patient diagnostic, therapeutic, rehabilitation, or monitoring and support needs.

Thus, in this study, we performed a qualitative literature review, focus group interviews (FGIs), and structured surveys to identify technologies and platforms useful for the management of older patients globally and the current healthcare systems for older patients in Korea. We then developed the Health-RESPECT (integrated caRE Systems for elderly PatiEnts using iCT) platform that focuses on the provision of care, support, and expert consultation to older adults admitted to $\mathrm{LTCH}$ or $\mathrm{NH}$ and requiring integrated care because of their multiple chronic diseases and functional decline.

\section{MATERIALS AND METHODS}

To develop the Health-RESPECT service model, we first conducted a qualitative literature review of existing ICT technologies or service models for the management of older adults and related research. Because research on ICT-based consultation or inter-professional relationships for chronic disease management is not sufficient, we included all types of research ranging from randomized controlled studies to observational studies. Under chronic diseases, we included diabetes and hypertension, which are the most prevalent diseases in older adults, and heart failure, which can be the most difficult to manage in long-term care facilities. The PubMed database was searched for relevant articles in English published in the last 10 years prior to the date of the search. The search strategies are shown in Supplementary Table S1.

We next conducted FGIs of medical staff, patients, and their family members. The interview focused on (1) difficulty in the management or care of older patients, (2) the current status of cooperative management between institutions, (3) the unmet needs for ICT-based integrated service models, (4) the specific content to be provided by ICT-based services, and (5) the requirements for the activation of ICT-based services.

On the basis of the results of FGIs, on- and off-line structured questionnaire surveys were designed and administered to measure quantified data from additional participants. The questions were organized to objectively describe the contents discussed in the FGI. The structured survey evaluated (1) the current status of cooperative management for older adults between institutions, (2) the current status and expected improvements in the use of comprehensive geriatric assessments (CGAs), and (3) comments on an ICT-based interdisciplinary service model for older adults. Because their major interests differed, medical staff (physicians/nurses) and patients/family members/caregivers were surveyed using different questions, and the data were analyzed separately.

On the basis of these findings, we developed the evidence-based Health-RESPECT, which included (1) CGA and customized management strategies, (2) a chronic disease management service including a decision support system, (3) a service to manage potentially inappropriate medications, (4) a tailored rehabilitation 
service, and (5) a consultation and videoconference service.

The study protocol was reviewed and approved by the Seoul National University Bundang Hospital Institutional Review Board (No. B-1908/556-304).

\section{RESULTS}

\section{Qualitative Literature Review}

To identify recent technology trends, platform development cases, and usability and effects of ICT-based chronic disease management in older adults, we reviewed a total of 60 articles ( 23 on hypertension, 18 on diabetes, and 19 on heart failure) indexed in the PubMed database. The rapid increase in research on the management of chronic diseases through ICT since 2009 has been attributed to technology development. The consultation systems mainly used web or mobile-based online platforms or single-application technologies. In most studies, both patients and medical staff participated. For hypertension and diabetes, ICT-based chronic disease management led to significant reductions in blood pressure or blood sugar levels. ${ }^{7-10)}$ In the case of heart failure, faster communication between patients and medical staff improved patient satisfaction and reduce hospitalized costs. ${ }^{11-13)}$ However, little research has been conducted on a consultation service model between the medical staff of different institutions (Supplementary Table S2, Fig. S1).

\section{Focus Group Interviews}

Between June 4 and 28, 2018, FGIs were conducted with 6 medical staff ( 2 acute care hospital physicians, 2 long-term care hospital physicians, 1 acute care hospital nurse, and 1 long-term care hospital nurse), 1 patient, 2 family members, and a hired caregiver. Most of the interviewees indicated that inter-institutional consultation is not well-established in Korea; however, due to complex multi-morbidities among older patients, there is a strong need for coordinated management between institutions. In addition, due to the absence of common geriatric evaluation tools, communication regarding functional status is impossible between institutions. Therefore, these FGIs underscored the importance of a common format for the comprehensive assessment of multi-morbidity, medication, nutrition, and functional status for efficient information sharing. In addition, the participants indicated that an online consultation system would be helpful for the management of duplicate drugs and chronic diseases. To activate the ICT-based, inter-professional consultation system, the physicians indicated the need for reimbursement for the invisible resource input provided by personnel. Meanwhile, patients or family members indicated that they would be willing to pay for an ICT-based consultation system that substituted for regular outpatient care without having to visit a university hospital or large center. They also expressed hope that the sharing of medical information between institutions would be more convenient with the ICT-based system.

\section{Structured Survey}

The FGI revealed the difficulties in evaluating and managing older patients while the qualitative literature review identified the usefulness of ICT-based management or consultation services in older patients. To collect and request more specific opinions and components to be included in the ICT-based management and consultation service system, we conducted an on- and off-line structured survey from August 16 to September 30, 2018, of 114 medical staff and 50 patients/family members/caregivers. Among the 114 medical staff ( $53 \%$ male) who participated in the structured survey, $80 \%$ were physicians and $20 \%$ were nurses; $45 \%$ belonged to tertiary hospitals and the others to LTCH and NH. Most medical staff $(94 \%)$ had over 1 year of experience in managing older adults. Among the 50 patients/family members/caregivers respondents, $76 \%$ were family members, $18 \%$ were hired caregivers, and $6 \%$ were patients.

The most difficult factors in the management or care of older patients were complex multi-morbidity ( $4.1 / 5$ points), absence of assessment tools and reimbursement systems for older patients (4.04/5), management of duplicate medications (3.96/5), lack of information about previous medical records (3.93/5), and lack of information about patient's pre-morbid function (3.90/5) (Fig. 1). The most common information transferred at the time of referral to other institutions was medical certificates and prescription and medical records; laboratory results or imaging files were relatively lower in necessity. The respondents most frequently reported using paper $(86.8 \%)$ to transfer medical information but reported being dissatisfied (2.6/5) with the amount, quality, and methodology. Medication and chronic disease management were among the services expected to be provided by the ICT-based management service. Among chronic diseases, the demand for management services was high in the order of diabetes, hypertension, and heart failure. In addition, as a management method, consultation on acute exacerbation, provision of the latest guidelines, and cumulative inquiry of results were preferred. However, the participants also expressed concerns about the lack of an adequate reimbursement system $(4.33 / 5)$ and leakage of private or medical information $(3.61 / 5)$. The types of rehabilitation services that most needed to be provided in the ICT-based service were swallowing, physical function, and cognitive rehabilitation.

Patients visited an average of 1.55 hospitals and met 2.66 physicians, with $42 \%$ having a history of transfer. The average travel time 


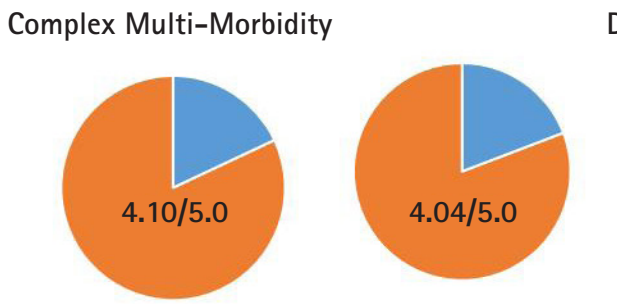

Absence of assessment tool
Duplicate medication

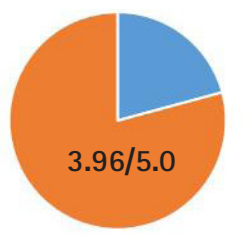

Unknown pre-morbid function
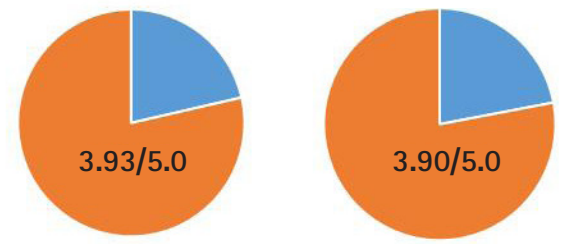

Lack of medical information

Fig. 1. Major difficulties in the management and care of older adults. The difficulties in the management or care of older patients included complex multi-morbidity (4.1/5 points), absence of assessment tools and reimbursement systems for older patients (4.04/5), duplicate medication management (3.96/5), lack of information about previous medical records $(3.93 / 5)$, and lack of information about patients' premorbid function $(3.90 / 5)$.

for outpatient/emergency visits was 1.72 hours, with an average cost of $\$ 41.22$ per person. Depending on the patient's place of care, the greatest cost and time was required for the transfer of older patients living in $\mathrm{NH}$ or LTCH. We observed a positive response that the sharing of medical records through the ICT-based service would be helpful for treatment $(4.16 / 5)$, prevention of duplicate prescription or drug abuse $(4.26 / 5)$, increased convenience of delivering medical information $(4.14 / 5)$, and money and time saving $(3.92 / 5)$. Similar to the medical staff survey results, there were also concerns about the leakage of private or medical information $(3.42 / 5)$. Regarding their willingness to pay for the ICT-based management and consultation system, we received above-average positive answers $(3.50 / 5)$ from patients, family members, and caregivers.

\section{Development of the Health-RESPECT}

The ICT-based management and consultation service model was developed to provide proper high-priority services for the medical staff of LTCH or NH. The detailed contents of the service model were identified through a literature review, FGIs, and structured surveys (Fig. 2).

Since the healthcare workers in LTCH in Korea assess patient general function, comorbidity status, and cognitive status monthly to claim specified daily fees for care service, we developed CGA based on these data. The medical information exchange system is used to transfer information on prescribed medications, diagnoses, laboratory data, and vital signs from the electronic medical records (EMRs) of LTCH to the service platform. The CGA integrates (1) existing medical information received from EMRs of LTCH and (2) additional evaluated data required for disease management or rehabilitation services. A CGA encompassing the 6 domains of comorbidity, physical function, swallowing function, cognitive function, activities of living, and medication was developed to be ad-

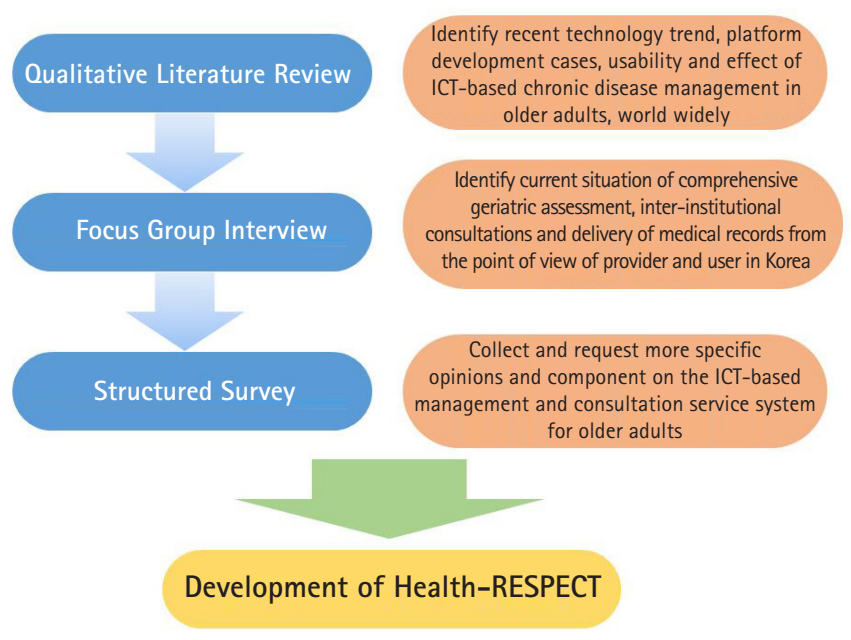

Fig. 2. Health-RESPECT development process. Qualitative literature review, focus group interview, and structured survey, as well as recent technology trends, inter-institutional consultations, and medical record delivery system identified the need for an information and communication technologies (ICT)-based system to manage older patients. The Health-RESPECT (integrated caRE Systems for elderly PatiEnts using iCT) was developed to reflect the requirements gathered in this process.

ministered to patients when they are initially included in the Health-RESPECT service platform. Physical function is evaluated by activities of daily living (ADLs) and instrumental ADLs (IADLs) with modified Barthel Index and Lawton and Brody Index. ${ }^{14,15)}$ Cognitive, swallowing, and physical functions are evaluated using the Korean version of the Mini-Mental State Examination (K-MMSE), the Standardized Swallowing Assessment (SSA), and the Functional Ambulatory Category (FAC), respectively. ${ }^{16-18)}$ Different versions of the MMSE (MMSE-K) used in long-term care facilities are substituted as needed. We used a validated self-report frailty questionnaire, based on the Korean version of the fatigue, re- 
sistance, ambulation, illnesses, and loss of weight scale (K-FRAIL). ${ }^{19)}$ On the basis of Beers criteria, 2016 SNUBH Inappropriate Medication for Elderly Criteria, and guidelines for chronic diseases, a list of drugs inappropriate for older adults was developed to fit for their clinical significance and frequencies of clinical use in $\mathrm{NH}$ and LTCH. $^{20-24)}$

On the basis of the results of the CGA, we also developed an individualized challenge list and treatment targets that included chronic disease management (hypertension, diabetes, and heart failure), drug management, and rehabilitation (cognitive, swallowing, and physical functions). The drug management service was developed by screening the medication currently prescribed and providing the number and identity of drugs matching those on the absolute or potentially inappropriate drug lists each month.

The treatment targets for chronic diseases were set according to the patient frailty status (Table 1). A chronic disease management service was developed to provide information about recommended and non-recommended combinations of medications, screening, responses to adverse events during treatment (orthostatic hypotension, hypoglycemia), drug adjustment according to renal function, comorbidity screening and management (diabetes-dyslipidemia), guidance for acute decompensated conditions, and lifestyle modifications based on recent guidelines. ${ }^{21-23)}$

In the rehabilitation service, video clips of exercise and swallowing rehabilitation of various levels are provided once weekly according to patients' physical and swallowing function, as evaluated by FAC or SSA. For example, patients who were non-functional ambulatory or ambulatory dependent on physical assistance (FAC $0-1$ ) are provided with videos of pressure sore prevention or sitting exercises through correct posture while patients who could ambulate independently are provided with videos of more intensive exercise. The cognitive rehabilitation program was developed for increasingly difficult orientation, attention, memory and problem-solving training in which patients with MMSE scores of 10 or more and 21 or less participated three times weekly.

The Health-RESPECT service has a system for issuing warning

Table 1. Treatment targets for hypertension and diabetes according to frailty status

\begin{tabular}{lccc}
\hline & Robust & Pre-frail & Frail \\
\hline Blood pressure (mmHg) & $140 / 90$ & $140 / 90$ & $150 / 90$ \\
Glycated hemoglobin $(\mathrm{HbAlc})(\%)$ & $<7.5$ & $<8.0$ & $<8.5$ \\
Random glucose level (mg/dL) & $\leq 190$ & $\leq 210$ & $\leq 230$ \\
\hline
\end{tabular}

Frailty status was evaluated using the Korean version of the Fatigue, Resistance, Ambulation, Illnesses, and Loss of Weight index (K-FRAIL). Scores of 3 and more, 1 to 2 , and 0 were classified as frail, pre-frail, and robust, respectively. alarms by message and written consultation service between institutions for vital or laboratory findings outside of the normal ranges or if the medical staff wish to do. Additionally, videoconferencing with acute care hospitals allowed regular management of patients living in LTCH or NH and participating in the Health-RESPECT service model (Fig. 3). Although the medical staff of long-term care facilities are responsible for primary decision-making, patients who may require changes in their assessment or prescription plans can be discussed bi-directionally through alarm messages, written consultations as needed or monthly video-conferencing.

To determine the expected clinical feasibility and usability of the various functions and tools included in the Health-RESPECT platform, we collected expert opinions from prospective users (medical staff from acute care hospital or long-term care facilities), who provided positive feedback, especially for the chronic disease and drug management tools. Additional corrections were made to the parts shown in these evaluations to be too complex or unusable.

\section{DISCUSSION}

This study identified the current status of the management or care for older patients in Korea through focus group interviews and a structured survey. In addition, we confirmed the necessity of an ICT-based platform for the exchange of medical information between institutions and for the treatment and care for older patients. The qualitative literature review identified that chronic disease management using ICT was also effective and efficient in older adults, leading to the development of the Health-RESPECT system with items and content suitable for older patients in Korea.

In Japan and Europe, where society aging has progressed further, government-supported policies and services actively use ICT to manage older populations in advance. ${ }^{25,26)}$ The use of ICT has allowed the development of service models and tools to support independent living, healthcare management through disease monitoring, fall detection, and emergency communication. For example, the DOREMI (Decrease of cOgnitive decline, malnutRition and sedEntariness by elderly empowerment in lifestyle Management and social Inclusion) project (Italy, 2013-2016) was developed to provide preventive strategies for senior citizens related to frailty, unhealthy nutrition, sedentariness, and cognitive decline. ${ }^{27)}$ The basis of the DOREMI environment is a context-aware and smart system able to learn and reason about older people, including their intentions, preferences, and purposes. Besides providing interventions, recording and monitoring information about the use of the system allows tracking of user performance over long periods, providing a potential alert to signs of malnutrition and physical and cognitive deterioration. 


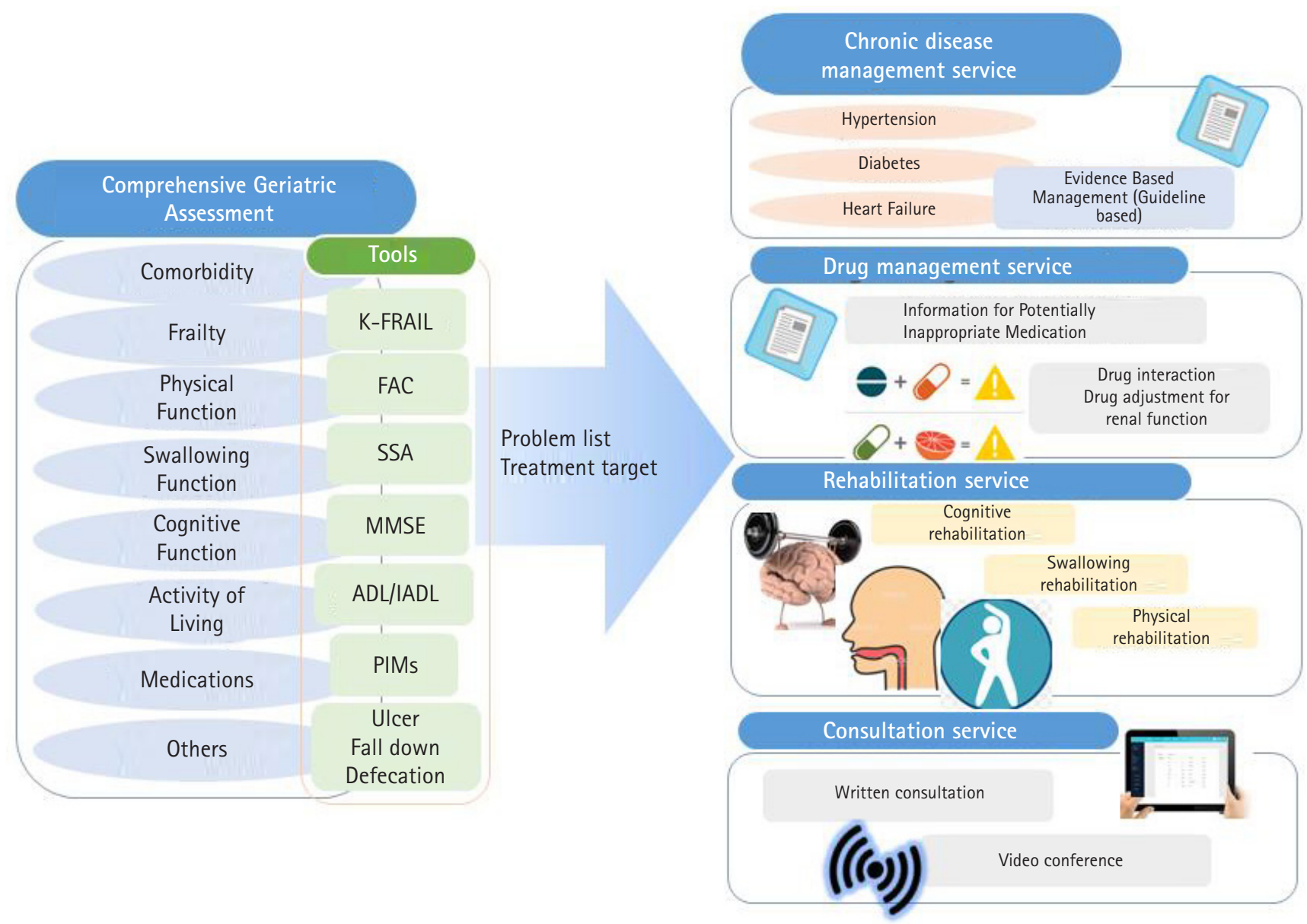

Fig. 3. Components of the Health-RESPECT program. We developed the Health-RESPECT (integrated caRE Systems for elderly PatiEnts using icT) system to provide tools for comprehensive geriatric assessment (CGA), individualized treatment strategies, chronic disease management, drug management, and rehabilitation programs. Systems for written consultation or videoconference allow for the management of acute exacerbation, aggravation, and patient transfer. K-FRAIL, Korean version of the fatigue, resistance, ambulation, illnesses, and loss of weight scale; FAC, Functional Ambulatory Category; SSA, Standardized Swallowing Assessment; MMSE, Mini-Mental State Examination; ADL, activities of daily living; IADL, instrumental activities of daily living; PIMs, potential inappropriate medications.

In Korea, many difficulties exist in the development and implementation of projects and the establishment of services to manage and care for older patients based on ICT. First, telemedicine, in which patients and doctors are not face-to-face, is legally prohibited in Korea. Therefore, we developed a usable ICT-based service platform for the management of older patients used by medical staff in LTCH and NH. The Health-RESPECT monitors for adverse events through an established algorithm and shared decisions can be made with distant professionals through consultation and videoconferencing. However, the system may also have a disadvantage in that additional effort and time may be required to use the new platform in addition to routine care. Approximately 30 minutes is required to perform CGA in new patients. Because of the nature of long-term care facilities, the patients have been hospitalized or institutionalized for long periods and the nurses in charge have a good under- standing of the patients' medical or functional condition. Moreover, medical staff are familiar with the test tools (MMSE or ADL) because these tests are periodically performed for reimbursement purposes. A pilot study determined that CGAs required an average of approximately 10 minutes to evaluate one patient. However, it would be hard to maintain this process without additional reimbursement. Therefore, we propose a pilot clinical study to determine appropriate reimbursement through evaluations of the clinical effectiveness and perform an economic analysis.

There is a growing need to support the development of diversified service models and systems using new technology to effectively treat and manage older patients and to help them lead independent lives in the community as members of society. ${ }^{28)}$ In this sense, this study is meaningful as it used ICT technology to develop a system to manage older patients living in LTCH and NH. Howev- 
er, as with most newly developed service models, the Health-RESPECT system requires validation through well-organized studies to provide evidence of its clinical effectiveness, utility, cost-effectiveness, safety, and the willingness of service users to pay.

\section{ACKNOWLEDGMENTS}

\section{CONFLICT OF INTEREST}

The researchers claim no conflicts of interest.

\section{FUNDING}

This work was supported by a grant from the Korea Health Technology R\&D Project through the Korea Health Industry Development Institute (KHIDI), funded by the Ministry of Health \& Welfare, Republic of Korea (No. HI18C0037)

\section{AUTHOR CONTRIBUTIONS}

Conceptualization: JYC, KIK, JYK, JIK, HK ML, SKJ, DHL, JL, YIJ; Data curation, CJY; Funding acquisition, KIK; Investigation, JYC, JYK, HK, ML, SKJ, YIJ, IHO; Methodology, JYC, JYK, YIJ; Project administration, JYC; Supervision, KIK, HK, JYK; Writing original draft, CJY, YIJ; Writing, review \& editing, KIK, HK.

\section{SUPPLEMENTARY MATERIALS}

Supplementary materials can be found via https://doi.org/10.4235/ agmr.20.0006

\section{REFERENCES}

1. Statistics Korea. Older people statistics in 2018 [Internet]. Daejeon, Korea: Statistics Korea; c2019 [cited 2020 Mar 11]. Available from: http://kostat.go.kr/portal/korea/kor_nw/1/1/index.board $?$ bmode $=$ read $\& a S e q=370779$.

2. Nihtila EK, Martikainen PT, Koskinen SV, Reunanen AR, Noro AM, Hakkinen UT. Chronic conditions and the risk of longterm institutionalization among older people. Eur J Public Health 2008; 18:77-84.

3. Park JM. Health status and health services utilization in elderly Koreans. Int J Equity Health 2014;13:73.

4. Akiyama N, Shiroiwa T, Fukuda T, Murashima S, Hayashida K. Healthcare costs for the elderly in Japan: analysis of medical care and long-term care claim records. PLoS One 2018; 13:e0190392.

5. European Commission. INclusive INtroduction of INtegrated CAre (IN3CA) [Internet]. Brussels, Belgium: European Commission; c2017 [cited 2020 Mar 11]. Available from: https:// cordis.europa.eu/project/id/621006.
6. Vollenbroek-Hutten M, Jansen-Kosterink S, Tabak M, Feletti LC, Zia G, N'dja A, et al. Possibilities of ICT-supported services in the clinical management of older adults. Aging Clin Exp Res 2017;29:49-57.

7. Saleh S, Farah A, Dimassi H, El Arnaout N, Constantin J, Osman $\mathrm{M}$, et al. Using mobile health to enhance outcomes of noncommunicable diseases care in rural settings and refugee camps: randomized controlled trial. JMIR Mhealth Uhealth 2018;6:e137.

8. Rinfret S, Lussier MT, Peirce A, Duhamel F, Cossette S, Lalonde $\mathrm{L}$, et al. The impact of a multidisciplinary information technology-supported program on blood pressure control in primary care. Circ Cardiovasc Qual Outcomes 2009;2:170-7.

9. Nilsson M, Rasmark U, Nordgren H, Hallberg P, Skonevik J, Westman G, et al. The physician at a distance: the use of videoconferencing in the treatment of patients with hypertension. J Telemed Telecare 2009; 15:397-403.

10. Cho JH, Lee HC, Lim DJ, Kwon HS, Yoon KH. Mobile communication using a mobile phone with a glucometer for glucose control in Type 2 patients with diabetes: as effective as an Internet-based glucose monitoring system. J Telemed Telecare 2009; 15:77-82.

11. Vuorinen AL, Leppanen J, Kaijanranta H, Kulju M, Helio T, van Gils M, et al. Use of home telemonitoring to support multidisciplinary care of heart failure patients in Finland: randomized controlled trial. J Med Internet Res 2014;16:e282.

12. Villani A, Malfatto G, Compare A, Della Rosa F, Bellardita L, Branzi G, et al. Clinical and psychological telemonitoring and telecare of high risk heart failure patients. J Telemed Telecare 2014;20:468-75.

13. Pisano F, Lorenzoni G, Sabato SS, Soriani N, Narraci O, Accogli $\mathrm{M}$, et al. Networking and data sharing reduces hospitalization cost of heart failure: the experience of GISC study. J Eval Clin Pract 2015;21:103-8.

14. Mahoney FI, Barthel DW. Functional evaluation: the Barthel Index. MD State Med J 1965;14:61-5.

15. Lawton MP, Brody EM. Assessment of older people: self-maintaining and instrumental activities of daily living. Gerontologist 1969;9:179-86.

16. Kang Y, Na DL, Hahn S. A validity study on the Korean Mini-Mental State Examination (K-MMSE) in dementia patients. J Korean Neurol Assoc, 1997;15:300-8.

17. Perry L. Screening swallowing function of patients with acute stroke. Part two: Detailed evaluation of the tool used by nurses. J Clin Nurs 2001; 10:474-81.

18. Mehrholz J, Wagner K, Rutte K, Meissner D, Pohl M. Predictive validity and responsiveness of the functional ambulation category in hemiparetic patients after stroke. Arch Phys Med Rehabil 
2007;88:1314-9.

19. Jung HW, Yoo HJ, Park SY, Kim SW, Choi JY, Yoon SJ, et al. The Korean version of the FRAIL scale: clinical feasibility and validity of assessing the frailty status of Korean elderly. Korean J Intern Med 2016;31:594-600.

20. By the 2019 American Geriatrics Society Beers Criteria Update Expert Panel. American Geriatrics Society 2019 updated AGS Beers Criteria for potentially inappropriate medication use in older adults. J Am Geriatr Soc 2019;67:674-94.

21. American Diabetes Association. Introduction: Standards of medical care in diabetes-2018. Diabetes Care 2018;41(Suppl 1):S1-S2.

22. Whelton PK, Carey RM, Aronow WS, et al. 2017 ACC/AHA/ AAPA/ABC/ACPM/AGS/APhA/ASH/ASPC/NMA/ PCNA Guideline for the prevention, detection, evaluation, and management of high blood pressure in adults: executive summary: a report of the American College of Cardiology/American Heart Association Task Force on Clinical Practice Guidelines. Hypertension 2018;71:1269-324.

23. Kim MS, Lee JH, Kim EJ, Park DG, Park SJ, Park JJ, et al. Korean guidelines for diagnosis and management of chronic heart failure. Korean Circ J 2017;47:555-643.
24. Park SJ, Choi NY, Suh YW, Lee JH, Lee ES, Lee SN et al. The effects of medication reconciliation on drug use and cost-saving by multidisciplinary geriatric care team. J Korean Soc Health Syst Pharm 2019;36:432-41.

25. Obi T, Ishmatova D, Iwasaki N. Promoting ICT innovations for the ageing population in Japan. Int J Med Inform 2013;82:e4762.

26. European Commission. Policies for ageing well with information and communication technologies (ICT) [Internet]. Brussels, Belgium: European Commission; c2019 [cited 2020 Mar 11]. Available from: https://ec.europa.eu/digital-single-market/en/ ageing-well.

27. The Community Research and Development Information Service (CORDIS). Decrease of cOgnitive decline, malnutRition and sedEntariness by elderly empowerment in lifestyle Management and social Inclusion [Internet]. Brussels, Belgium: European Commission; c2017 [cited 2020 Mar 11]. Available from: https://cordis.europa.eu/project/id/611650.

28. Kim KI. Optimal management of chronic medical conditions through digital medicine. Ann Geriatr Med Res 2018;22:11720. 